\title{
Gut immune dysfunction through impaired innate pattern recognition receptor expression and gut microbiota dysbiosis in chronic SIV infection
}

\author{
TW Glavan ${ }^{1,5}$, CA Gaulke ${ }^{1,5,6}$, C Santos Rocha $^{1}$, S Sankaran-Walters $^{1}$, LA Hirao ${ }^{1}$, M Raffatellu ${ }^{2}$, G Jiang $^{1}$,
} AJ Bäumler ${ }^{1}$, LR Goulart ${ }^{1,3}$ and S Dandekar ${ }^{1,4}$

HIV targets the gut mucosa early in infection, causing immune and epithelial barrier dysfunction and disease progression. However, gut mucosal sensing and innate immune signaling through mucosal pattern recognition receptors (PRRs) during HIV infection and disease progression are not well defined. Using the simian immunodeficiency virus (SIV)-infected rhesus macaque model of AIDS, we found a robust increase in PRRs and inflammatory cytokine gene expression during the acute SIV infection in both peripheral blood and gut mucosa, coinciding with viral replication. PRR expression remained elevated in peripheral blood following the transition to chronic SIV infection. In contrast, massive dampening of PRR expression was detected in the gut mucosa, despite the presence of detectable viral loads.

Exceptionally, expression of Toll-like receptor 4 (TLR4) and TLR8 was downmodulated and diverged from expression patterns for most other TLRs in the gut. Decreased mucosal PRR expression was associated with increased abundance of several pathogenic bacterial taxa, including Pasteurellaceae members, Aggregatibacter and Actinobacillus, and Mycoplasmataceae family. Early antiretroviral therapy led to viral suppression but only partial maintenance of gut PRRs and cytokine gene expression. In summary, SIV infection dampens mucosal innate immunity through PRR dysregulation and may promote immune activation, gut microbiota changes, and ineffective viral clearance.

\section{INTRODUCTION}

The gut-associated lymphoid tissue is an important early site of HIV replication and severe mucosal $\mathrm{CD} 4{ }^{+}$T-cell depletion. ${ }^{1-4}$ A stable viral reservoir is established in the gut very early in infection that is not eradicated even during long-term suppressive antiretroviral therapy (ART). ${ }^{2,5}$ The loss of mucosal $\mathrm{CD}^{+}{ }^{+} \mathrm{T}$ cells, altered T-cell homeostasis, and epithelial barrier disruption are linked to microbial translocation, chronic immune activation, and disease progression. ${ }^{2,6,7}$ However, changes in the gut microenvironment during early HIV infection are not adequately reflected in the peripheral blood, highlighting the importance of the gut mucosal assessments to investigate pathogenic mechanisms.
Chronic HIV infection is characterized by persistent viral replication, unresolved immune activation, and progressive decline of immune function. ${ }^{2,5,6,8}$ Despite effective suppression of viral replication during long-term ART, it fails to eradicate viral reservoirs and fully resolve chronic inflammation. ${ }^{2,6,9,10}$ Increased inflammatory cytokine production and altered gut microbiota are reflective of pathogenic effects of chronic HIV infection. ${ }^{11-13}$ Signaling through pattern recognition receptors (PRRs) in the gastrointestinal tract drives the sensing of pathogens and initiation of innate inflammatory immune responses while maintaining immune tolerance to resident gut microbiota. Therefore, it is possible that PRR expression and signaling may be exploited by pathogens to invade the gut mucosa. Conversely, the host may modulate PRR expression to

\footnotetext{
${ }^{1}$ Department of Medical Microbiology and Immunology, University of California, Davis, California, USA. ${ }^{2}$ Department of Microbiology and Molecular Genetics, University of California, Irvine, California, USA. ${ }^{3}$ Institute of Genetics and Biochemistry, Federal University of Uberlandia, Uberlandia, Brazil and ${ }^{4}$ California National Primate Research Center, Davis, California, USA. Correspondence: S Dandekar (sdandekar@ucdavis.edu)

${ }^{5}$ These authors contributed equally to this work.

${ }^{6}$ Current address: Department of Microbiology, Oregon State University, Corvallis, Oregon 97331, USA.
} 
limit the pathogen-driven inflammation and damage to the gut microenvironment. Current understanding is limited on whether HIV infection impairs PRR expression and innate immune response at the gut mucosal surfaces.

The human gut microbiota has an essential role in maintaining immune homeostasis. In addition to providing a physical barrier against outgrowth of pathogenic bacteria, components of the gut microbiota aid in digestion of nutrients, produce important factors that support epithelial growth and barrier function, and guide the development of the immune system. ${ }^{14}$ Changes in the composition and diversity of gut microbiota have been linked to inflammation and disease. ${ }^{15}$ Disruption of the gut microbiota involving increased microbial diversity and increased presence of potentially pathogenic bacterial families is reported in HIV-infected patients and in SIV-infected non-human primates. ${ }^{11-13,16-19}$ These studies primarily focused on the lower gastrointestinal tract and oral cavity. However, shifts in microbiota inhabiting the small intestine engaged in nutrient digestion and absorption have been under-investigated. Moreover, it is not known whether SIV/HIV infection modulates changes in the mucosal expression of receptors that sense microbial products, including PRRs, and whether infection-associated changes in PRR expression influence aberrant production of inflammatory cytokines as well as gut microbiota composition.

Using the SIV model of AIDS, we found a robust increase in the expression of multiple PRRs and associated cytokines in the gut mucosa during early SIV infection that was followed by a remarkable dampening of PRR and cytokine gene expression during therapy-naive chronic viral infection, despite the persistence of high viral loads. In contrast, increased PRR expression was maintained in peripheral blood at all stages of infection. The exception was for Toll-like receptors (TLRs) 4 and 8 whose expression was downmodulated or unchanged during SIV infection. Altered PRR expression in SIV infection was associated with changes in microbiome diversity and increased immune activation. An early initiation of ART resulted in elevated levels of PRR expression and enhanced immune recovery. Our findings suggest that modulation of PRR signaling during viral infection may be linked to incomplete viral clearance, increased immune activation, and decreased microbiome diversity. Therefore, PRR signaling and/or microbiota modulation in the gut may be beneficial therapeutic targets to reverse the pathological consequences of HIV infection.

\section{RESULTS \\ Viral replication and $\mathrm{CD} 4^{+} \mathrm{T}$-cell depletion in peripheral blood and gut mucosa during SIV infection}

Viral RNA loads were detected in both peripheral blood and jejunum samples during acute and chronic stages of pathogenic SIVmac251 infection (see Supplementary Table S1 online and Figure 1a). Viral loads averaged $2.8 \times 10^{6}$ copies per ml plasma and $1.5 \times 10^{5}$ copies per $\mu \mathrm{g}$ total RNA from jejunum tissue during acute SIV infection. Average viral loads were reduced to $4.4 \times 10^{4}$ copies per ml in plasma and to $10^{4}$ copies per $\mu \mathrm{g}$ total
RNA in jejunum tissues during chronic SIV infection (Figure 1a, $P<0.01$ compared with acute infection). Severe $\mathrm{CD} 4{ }^{+} \mathrm{T}$-cell loss was consistently detected in the gut mucosa $\left(9.1 \pm 8.4 \% \mathrm{CD}^{+}{ }^{+} \mathrm{CD} 4{ }^{+}\right.$cells) during acute SIV infection that persisted $(4.3 \pm 4.1 \%)$ through chronic viral infection (Figure 1c). $\mathrm{CD}^{+} \mathrm{T}$-cell loss in peripheral blood was progressive in acute $(35.8 \pm 10.0 \%)$ and in chronic infection $(31.8 \pm 19.0 \%)$, compared with uninfected controls $(52.2 \%)$. As previously reported, ${ }^{20}$ early initiation of ART ( 1 week post-SIV infection) resulted in a remarkable decrease in viral loads in both plasma and jejunum samples and $>80 \%$ of CD $4+$ T-cell restoration (see Supplementary Table S1). Despite having plasma viral loads below detection limits, residual viral replication was detected in the gut.

SIVmac1A11, a non-pathogenic viral strain, successfully establishes infection in the host but does not cause immunodeficiency. ${ }^{21,22}$ Experimental SIVmac1A11 infection led to low levels of viral replication (average $1.9 \times 10^{3}$ copies per $\mu \mathrm{g}$ total RNA) in the jejunum during acute viral infection (Figure 1b). They are significantly lower $(\sim 3 \log$ difference) compared with those with pathogenic SIVmac251 infection $(P<0.05)$ (Figure 1a). In chronic SIVmac1A11 infection, all but one animal had viral loads below the limit of detection.

Increased expression of PRRs in peripheral blood during We sought to determine whether PRR expression was altered in the peripheral blood during the acute and chronic stages of SIV infection. A significant increase in the expression of TLRs 1, 2, 6,7 , and $10(P<0.05)$ was seen during chronic SIV infection, as compared with uninfected controls (Figure 2a). Expression of cytosolic sensors RIG-I and MDA5 was not significantly altered (Figure 2a). Thus chronic SIV infection resulted in increased TLR expression in peripheral blood mononuclear cells (PBMCs).

\section{Marked increase in PRR expression in the gut during acute SIV infection followed by massive dampening during chronic infection}

We investigated changes in the expression of PRRs in the gut mucosa during SIV infection. A robust increase in the expression of MDA-5 and TLRs 2, 5, 6, 7, 9, and $10(P<0.05)$ was detected in intestinal tissue during acute SIV infection (Figure 2b). These changes mirrored the increased TLR expression observed in peripheral blood samples (Figure 2a). However, the expression of these PRRs was substantially higher in the gut than in peripheral blood (Figure 2a,b). Further, robust expression of TLRs 5 and 9 was seen only in the gut mucosal tissues but not in PBMCs of SIV-infected animals compared with uninfected controls.

During chronic SIV infection, a remarkable dampening of PRRs expression $(P<0.05$; TLRs $2,6,7,9,10)$ was observed in gut mucosal tissues, when compared with those in acute SIV infection (Figure 2b). The dampening of PRR expression occurred despite the persistence of viral loads in the gut (Figure 1a). Among TLRs, endosomal TLR3, TLR7, and TLR9 are known to detect HIV and SIV nucleic acids. 
a

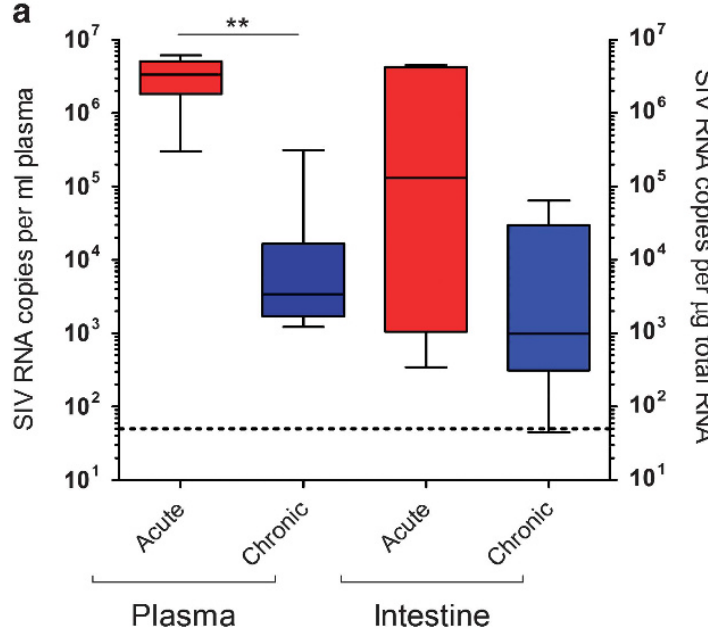

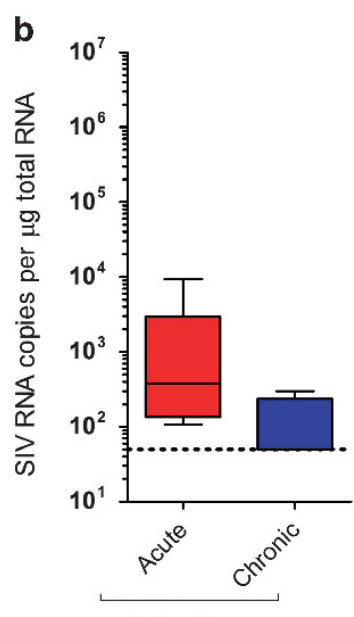

Intestine

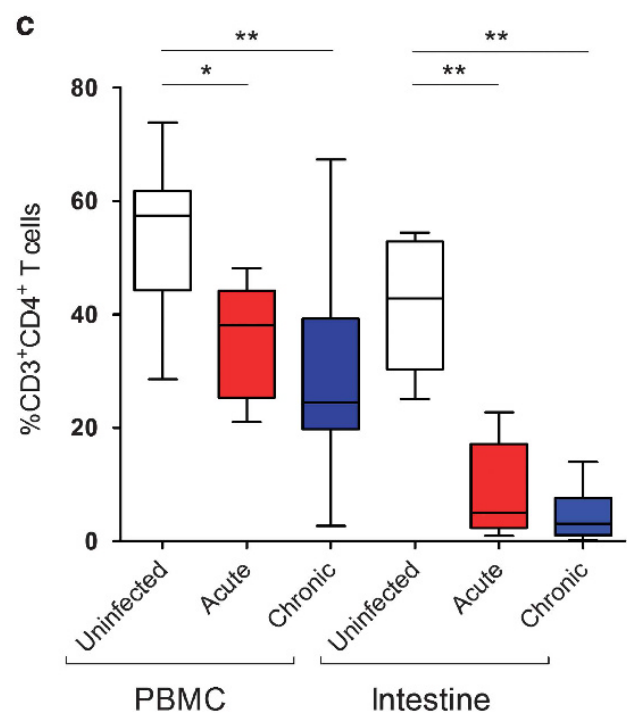

Figure 1 Viral loads and CD4 + T-cell depletion in peripheral blood and gut mucosal compartments during simian immunodeficiency virus (SIV) infection. Viral RNA loads were measured in the plasma and gut tissue during acute and chronic stages of (a) pathogenic SIVmac251 and (b) nonpathogenic SIVmac1A11 infection by quantitative real-time PCR. (c) Percentages of CD ${ }^{+} \mathrm{CD} 4{ }^{+} \mathrm{T}$ cells were determined by flow cytometry. Statistical analysis was performed by comparison with uninfected controls. ${ }^{\star} P<0.05 ;{ }^{\star \star} P<0.01$.

Expression levels of TLR7 and 9 genes were markedly increased in the gut during acute infection but decreased to baseline levels during chronic infection. In summary, there is discordance between peripheral blood and gut mucosal compartments for PRR expression, which was most pronounced during chronic SIV infection.

Although majority of the TLRs had a striking increase in their expression levels in the gut and peripheral blood, TLR4 and TLR8 had a divergent expression pattern. No increase in TLR4 or TLR8 expression in the gut was observed during acute as well as chronic stages of SIV infection (Figure 2b). This is surprising considering the presence of viral RNA and antigens, as well as, active viral replication in the gut. There was a trend of decreased TLR4 expression during the acute SIV infection although the values did not reach statistical significance. Decreased expression was also observed for TLR4 and TLR8 during the acute SIV infection in peripheral blood (Figure 2a).

\section{Preservation of PRR expression in the gut following the early initiation of ART}

We found that early initiation of ART during HIV and SIV infection can suppress viral replication and restore $\mathrm{CD} 4{ }^{+} \mathrm{T}$ cells in the gut mucosa. ${ }^{6,20,23}$ In order to determine the effects of therapeutic intervention on the restoration of gut innate immune homeostasis, we measured the PRR gene expression in jejunum samples from animals that received ART during acute SIV infection. As previously reported, the early initiation of ART (at 1 week post-SIV infection) led to suppression of viral RNA loads to very low to undetectable levels and resulted in $>80 \%$ restoration of $\mathrm{CD}^{+}{ }^{+} \mathrm{T}$-cells in the gut and nearly to preinfection levels in the peripheral blood compared with animals initiating ART during chronic SIV infection and therapy-naive SIV infected controls. ${ }^{20}$ Early ART partially prevented the dampening of the PRR expression in gut tissues of these animals (Figure 2b). The PRR expression profile 
a

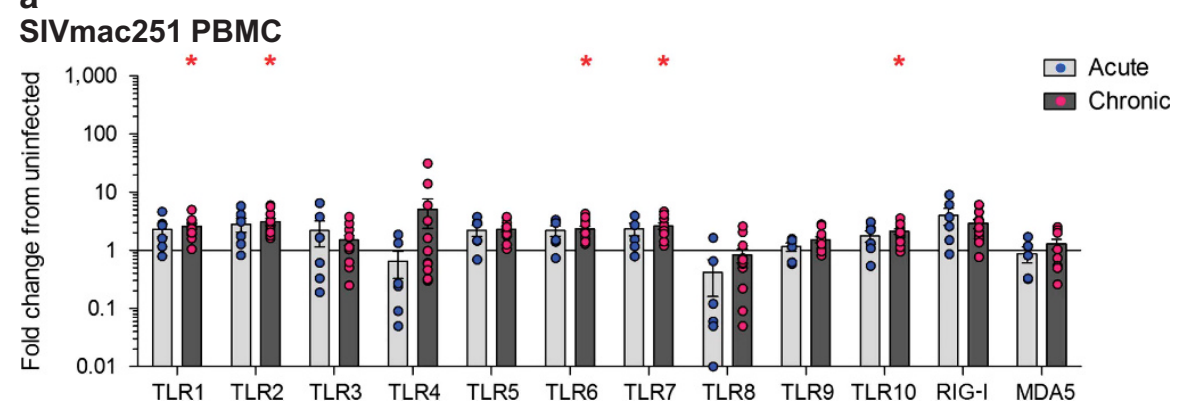

b

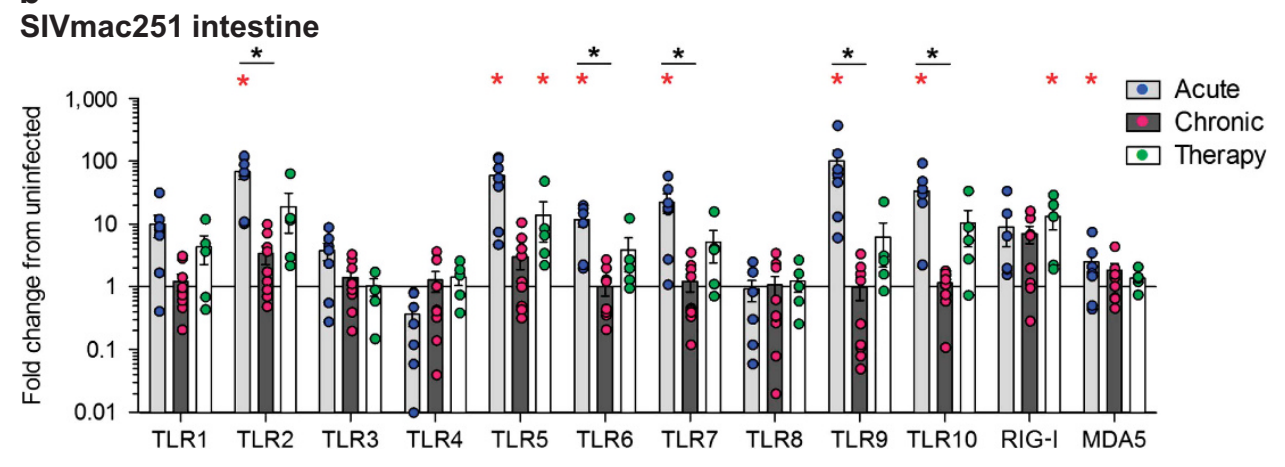

C
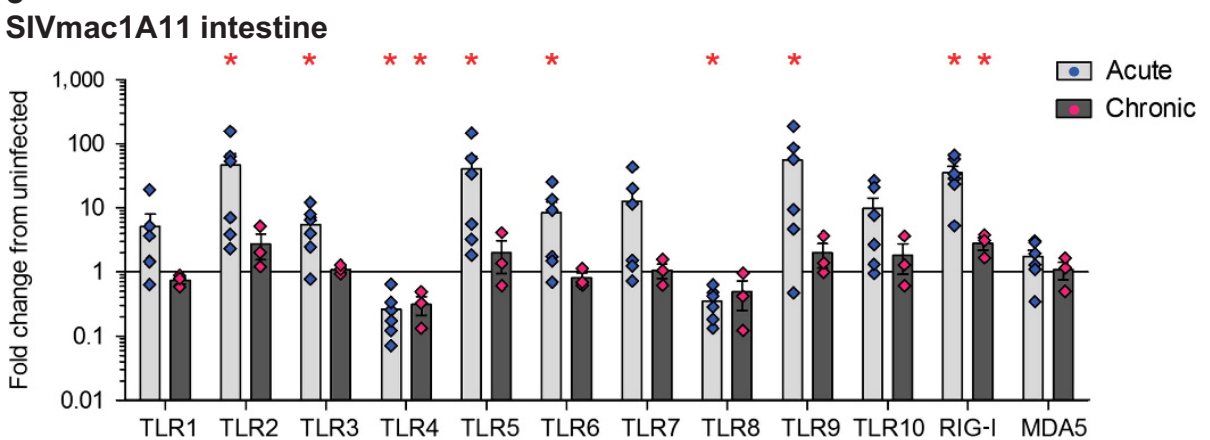

Figure 2 Expression profiles of pattern recognition receptors (PRRs) during acute and chronic stages of simian immunodeficiency virus (SIV). The PRR gene expression was determined by quantitative real-time PCR in the (a) peripheral blood mononuclear cells (PBMCs) and (b) gut tissue during acute or chronic (therapy-naive or with antiretroviral therapy) stages of SIVmac251 infection compared with SIV-negative healthy controls. (c) The PRR expression was measured in gut samples during the acute and chronic stages of non-pathogenic SIVmac1A11 infection. Data are shown as the average fold changes compared with uninfected controls. ${ }^{*} P<0.05$ (red asterisk), compared with uninfected control animals; ${ }^{*} P<0.05$ (black asterisk), compared with chronically infected animals. TLR, Toll-like receptor.

during therapy was similar to that observed during acute SIV infection. These data suggest that early ART was able to partially restore the mucosal sensing and response through PRR expression, and this coincided with the suppression of viral loads and restoration of mucosal $\mathrm{CD} 4+\mathrm{T}$ cells.

\section{Preservation of PRR expression in animals with non-pathogenic SIV1A11 infection}

We sought to determine whether dampening of the PRR expression in the gut during chronic SIV infection was related to viral loads and pathogenicity of the virus. We determined PRR expression in animals infected with non-pathogenic SIVmac1A11. ${ }^{21}$
An increase in the PRR expression was detected during acute SIVmac1A11 infection and the trend was similar to that during acute pathogenic SIV infection. A significant increase in the expression of TLRs 2, 3, 4, 5, 6, 9, and RIG-I was detected in SIVmac1A11-infected animals, compared with uninfected controls $(P<0.05$, Figure 2c). During chronic SIVmac1A11 infection, PRR expression decreased to the levels comparable to those seen in SIV-negative controls (Figure 2c). This decline coincided with an effective suppression of viral replication in the gut mucosa, suggesting that increased TLR expression during acute viral infection was in response to viral loads. These findings are in contrast to our findings from pathogenic chronic SIVmac251 infection that showed decreased gut mucosal PRR expression in spite of persistent viral replication and detectable 
viral loads, further validating the dysregulation of mucosal TLR expression during pathogenic SIV infection (Figure $\mathbf{2 b}, \mathbf{c}$ ).

PRR expression of RIG-I or TLR3 negatively correlates with SIV replication

To further investigate whether expression of PRR has a role in the suppression of viral replication, we performed correlation analyses. Among these PRRs, only expression of RIG-I or TLR3 displayed a significant negative correlation with
SIVmac251 loads (Figure 3a). Both viral nucleic acid sensors RIG-I and TLR3 were previously shown to inhibit HIV or SIV replication. ${ }^{24,25}$ Rapid induction of RIG-I and TLR3 during acute SIV infection suggests that the virus was detected by these PRRs. In contrast, there was no correlation of any PRRs, including RIG-I or TLR3, with SIVmac1A11 replication (Figure 3b), indicating that induction of RIG-I or TLR3 during acute SIVmac1A11 infection might have partly contributed to effective suppression of SIVmac1A11 replication. a
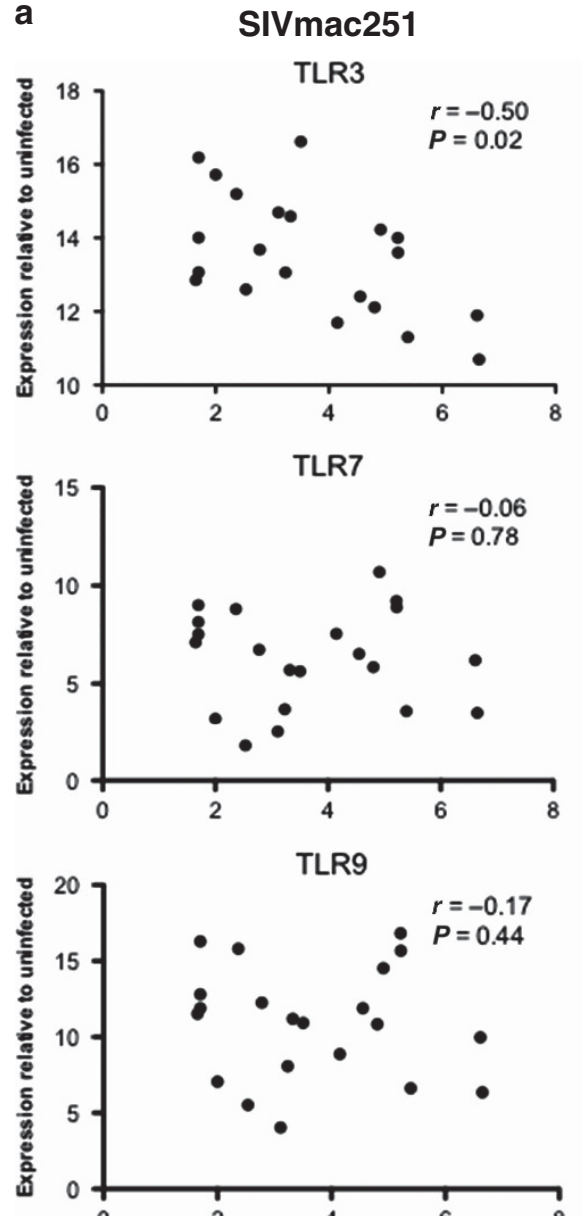

RIG-I

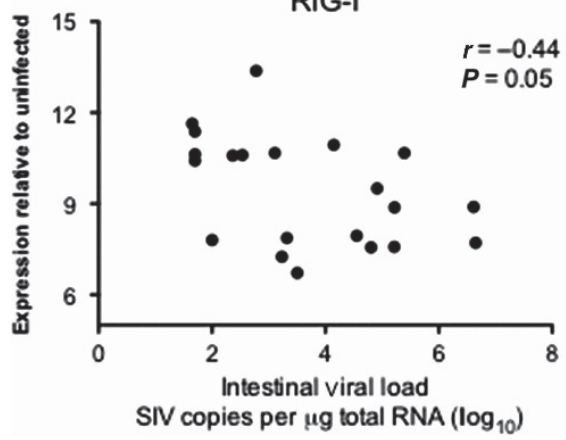

b
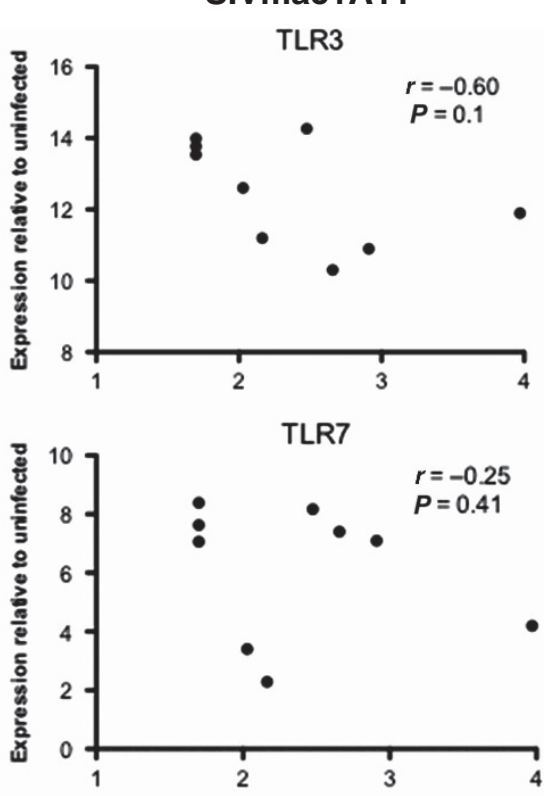

TLR9

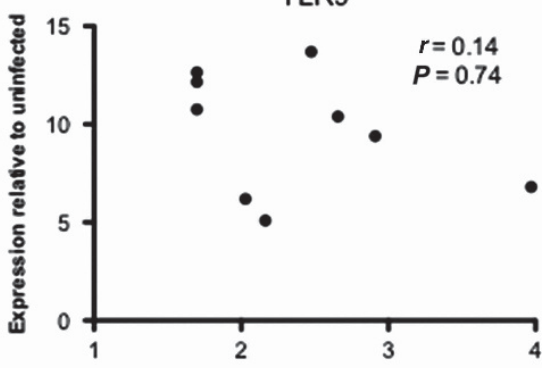

RIG-I

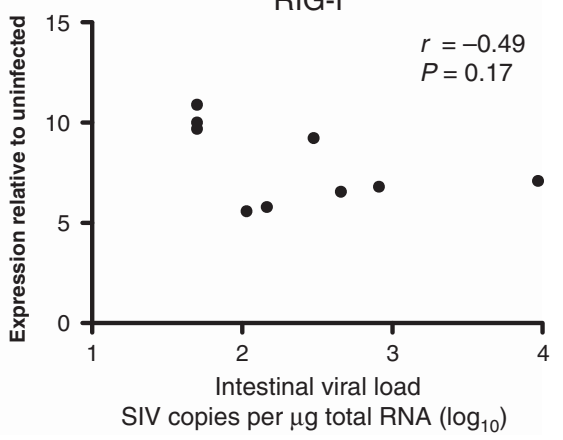

Figure 3 A correlation of pattern recognition receptor expression with viral replication during acute simian immunodeficiency virus (SIV) infection. Spearman rank correlations were performed between expression levels of Toll-like receptor 3 (TLR3), TLR7, TLR9, and RIG-I and intestinal viral loads from (a) SIVmac251- and (b) SIVmac1A11-infected animals. 
Inflammatory cytokine expression reflects changes in PRR expression during SIV infection

To determine whether changes in PRR expression during SIV infection were also accompanied by associated mucosal responses, we measured the expression of inflammatory and immune-modulatory cytokines. There was a significant increase $(P<0.05)$ in the expression of the pro-inflammatory cytokines tumor necrosis factor $\alpha$ (TNF $\alpha$ ), interleukin (IL)-12, IL-8, and IL-22 in the peripheral blood (Figure 4a) and interferon- $\alpha$ (IFN $\alpha)$, IFN $\gamma, \mathrm{TNF} \alpha, \mathrm{IL}-12, \mathrm{IL}-8, \mathrm{IL}-17, \mathrm{IL}-22$, and IL-23 in the jejunum compartments (Figure 4b) of animals with acute SIV infection, as compared with uninfected controls. The magnitude of the induction of inflammatory cytokines in the gut mucosa was substantially greater than that seen in peripheral blood.

Cytokines induced during acute SIV infection (IFN $\gamma, \operatorname{IFN} \alpha$, TNF $\alpha$, IL-17, IL-12, IL-8, and IL-22) were significantly downmodulated in the gut during the transition from acute to chronic SIV infection. Nevertheless, IL-8, IFN $\gamma$, and IL-22 levels remained elevated compared with SIV-negative controls (Figure 4b). In contrast to the gut, the expression of cytokines remained elevated in the peripheral blood during both acute and chronic SIV infection (Figure 4a). Initiation of ART during early SIV infection resulted in the control of viral replication and an elevated level of several cytokines involved in immune response.

a

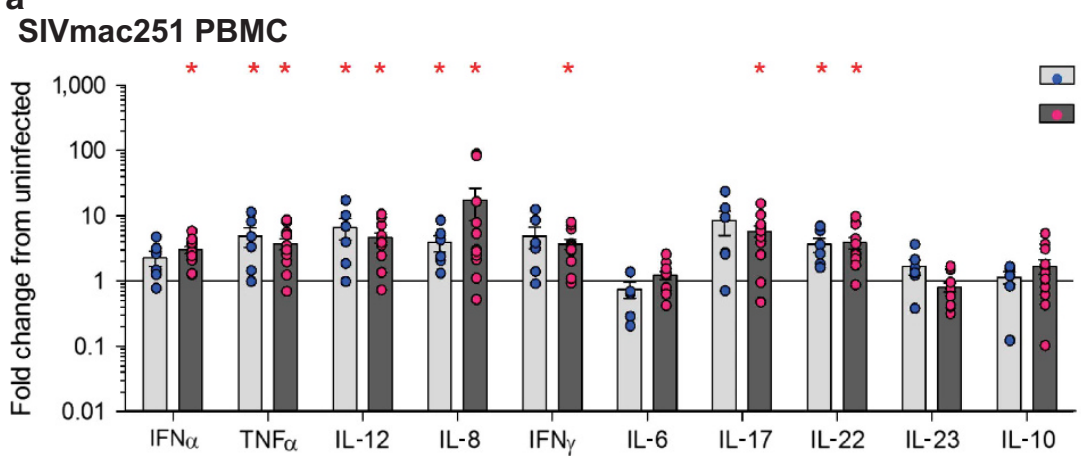

b
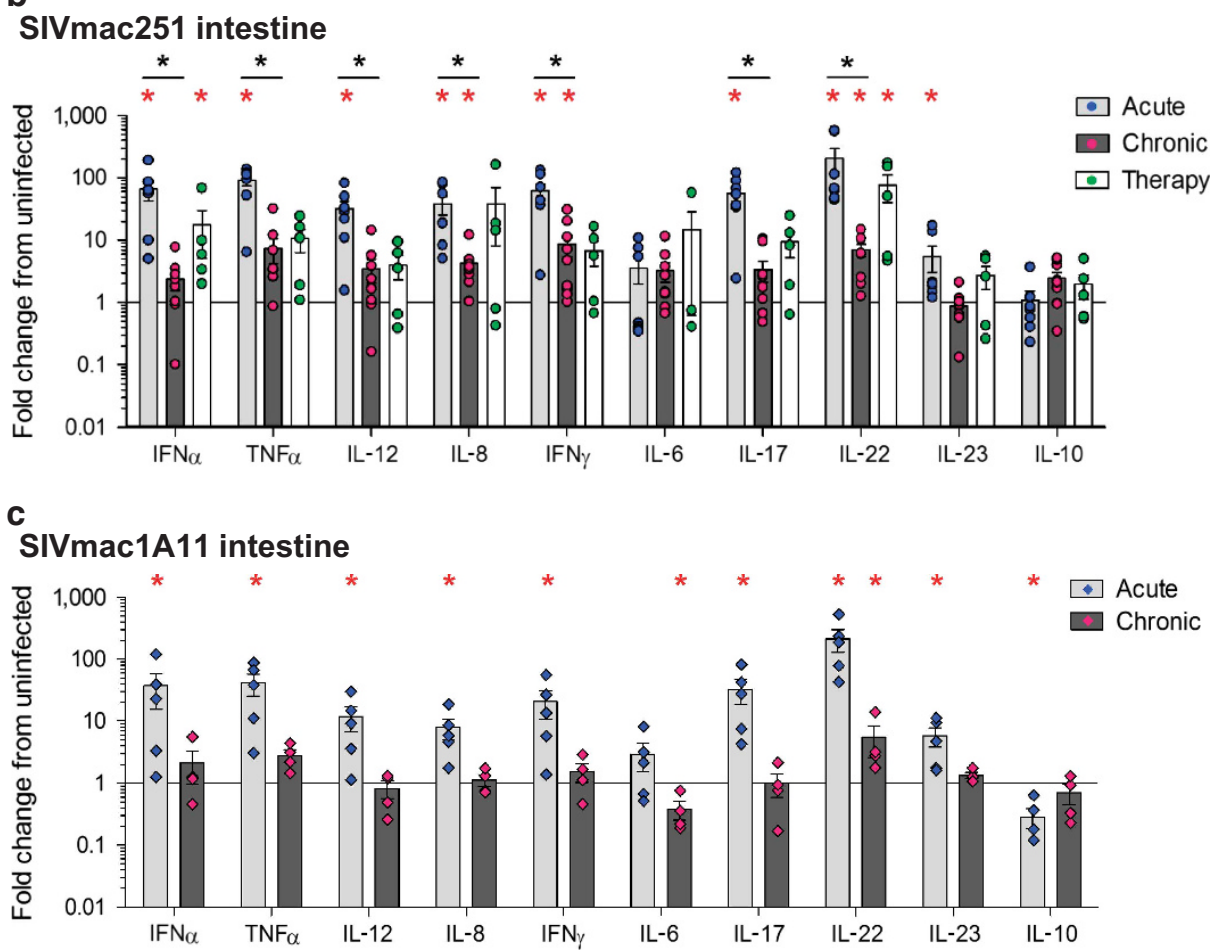

Figure 4 Changes in the cytokine expression during acute and chronic stages of simian immunodeficiency virus (SIV) infection. Cytokine gene expression was analyzed in the (a) peripheral blood mononuclear cells (PBMCs) and (b) gut tissue during acute and chronic (therapy-naive or with antiretroviral therapy) stages of pathogenic SIV infection compared with SIV-negative controls using quantitative real-time PCR. (c) Cytokine gene expression was measured in the gut tissue during acute and chronic stages of non-pathogenic SIVmac1A11 infection. The data are shown as an average fold change compared with SIV-negative controls. ${ }^{\star} P<0.05$ (red asterisk), compared with uninfected control animals; ${ }^{*} P<0.05$ (black asterisk), compared with chronically infected animals. IFN, interferon; IL, interleukin; TNF, tumor necrosis factor. 
Expression of pro-inflammatory cytokines was also increased in non-pathogenic SIVmac1A11-infected animals during acute infection and was similar to the profile seen during SIVmac251 infection (Figure $\mathbf{4 b}, \mathbf{c}$ ). However, cytokine expression was reduced to basal levels during chronic SIV1A11 infection that was comparable to uninfected controls. No demonstrable change in the expression of anti-inflammatory cytokine IL-10 was seen during acute or chronic SIVmac251 infection while IL-10 expression was elevated during chronic SIV1A11 infection. Collectively, these data indicate that the elevated PPR expression induced during SIV infection was associated with inflammatory cytokine expression and that early ART did not decrease levels of both PRR and inflammatory cytokines to the basal control levels in the gut mucosa, possibly owing to the residual viral replication.

\section{SIV infection leads to changes in the gut microbiota}

Recent studies have reported alterations in the composition and complexity of gut microbial communities during chronic HIV and SIV infections. ${ }^{11-13,16-18}$ Based on the differential kinetics of PRR and cytokine expression in intestinal mucosa during SIV infection, we sought to investigate whether the pro-inflammatory environment during SIV infection would cause alterations in the gut microbiota from the same sites of PRR and cytokine analyses. In contrast, most of the previous studies of gut microbiota during HIV and SIV infections evaluated fecal samples. To determine the changes occurring in the gut microbiota during SIV infection, we constructed and sequenced 16S rRNA amplicon libraries from gut tissue of SIV-infected animals and healthy SIV-negative controls. Gut microbial communities differed from one animal to another (Figure 5a). Microbiota from jejunum samples of all the animals predominantly consisted of three phyla: Firmicutes, Bacteroidetes, and Proteobacteria (Figure 5a). The most notable change during acute SIV infection was a significant increase in the levels of Proteobacteria (Figure 5b). A decrease in Firmicutes abundance was also detected during both acute and chronic SIV infection although not statistically significant (Figure 5b). The abundance of Bacteroidetes was comparable between SIV-negative controls and SIV-infected animals, and there was no detectable difference in the remaining phyla.

Few changes were observed at lower bacterial taxonomic levels. Within the Proteobacteria phylum, Pasteurellaceae family members, including Aggregatibacter and Actinobacillus, became more abundant during acute SIV infection and then significantly less abundant during the chronic viral infection (Figure 6). A significant increase in the abundance of Mycoplasmataceae, a family within the Tenericutes phylum, was also detected during chronic infection compared with uninfected animals (Figure 6). No significant changes were observed within other phyla. However, there was a decreased trend $(P=0.08)$ for Streptococcus abundance during acute and chronic infection and for increased abundance of Staphylococcus only in the chronic SIV infection that approached significance (Figure 6).

\section{Alterations in gut microbiota correlate with aberrant cytokine and PRR expression}

To investigate whether changes in the microbiome of SIV-infected animals could be directly associated with expression of specific PRRs or cytokines, we examined correlations between the most abundant operational taxonomic units ( $>0.5 \%$ in all samples) and expression of PRR and cytokine genes during SIV infection (Figure 7a). We found that
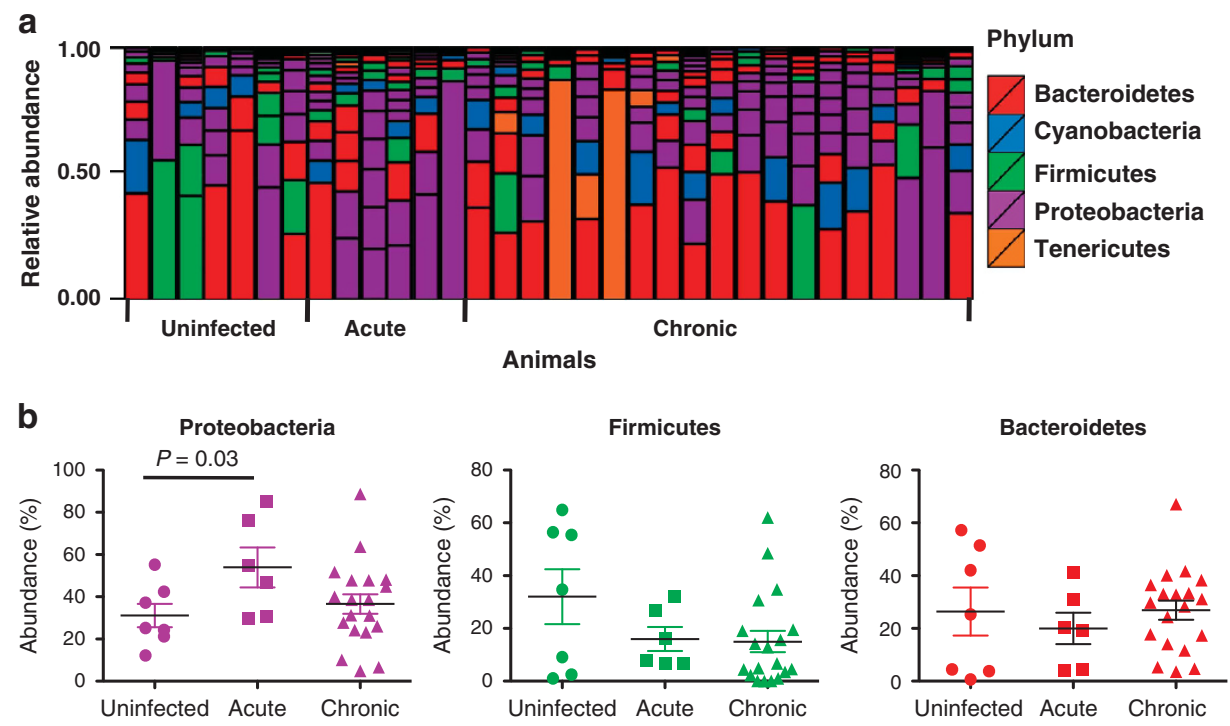

Figure 5 Simian immunodeficiency virus (SIV) infection induced changes in the gut microbiota. The gut microbiota from jejunum tissue samples of SIV-infected macaques were sequenced for $16 \mathrm{~S}$ amplicons. Data from animals during the acute $(n=6)$ and chronic SIV infection $(n=19)$ were compared with those from SIV-negative healthy controls $(n=7)$. (a) Bar charts represent the group average percentage of the total filtered reads that aligned to the indicated phylum. (b) Relative abundance of Proteobacteria, Bacteroidetes, and Firmicutes in SIV-negative and SIV-infected macaques during acute and chronic stages of infection. 

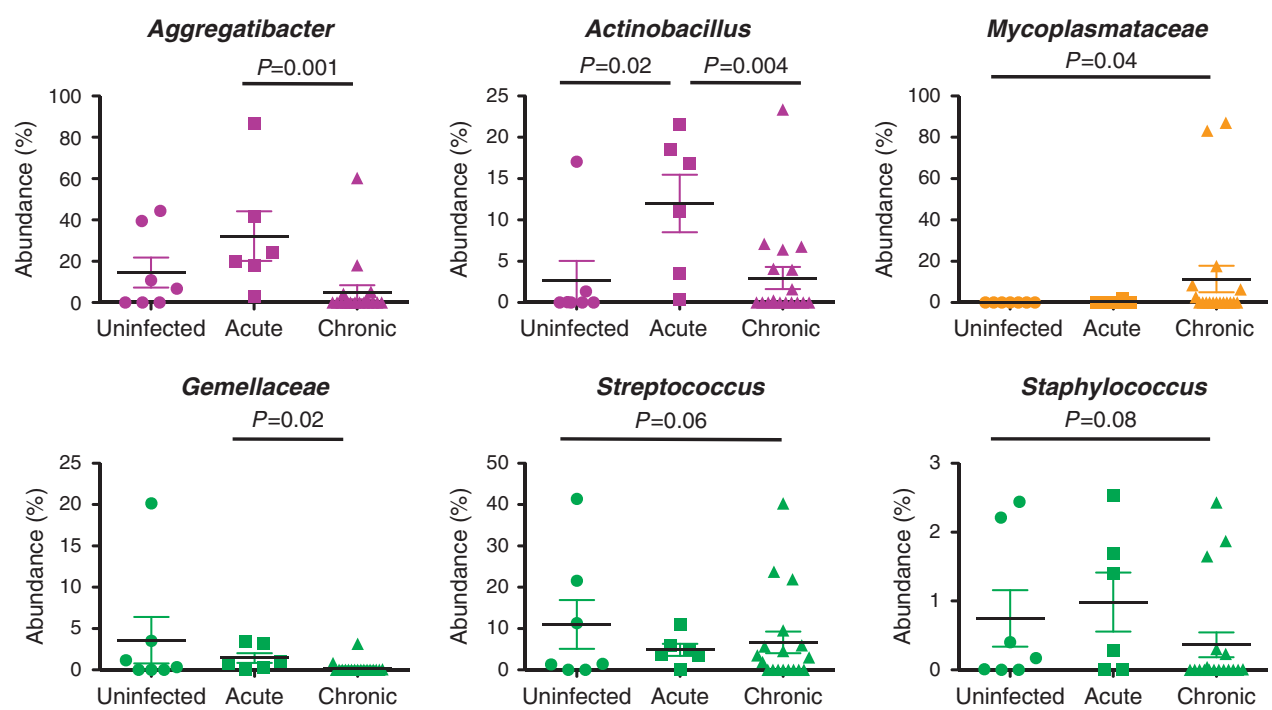

Figure 6 An increased abundance of potentially pathogenic microbial taxa during simian immunodeficiency virus (SIV) infection. Dot plots show relative abundance of Aggregatibacter, Actinobacillus, Mycoplasmataceae, Gemellaceae, Streptococcus, and Staphyclococcus in SIV-negative and SIV-infected animals. Significant differences between groups were determined using non-parametric Mann-Whitney $U$-tests $(P$-value $\leqslant 0.05)$.

a

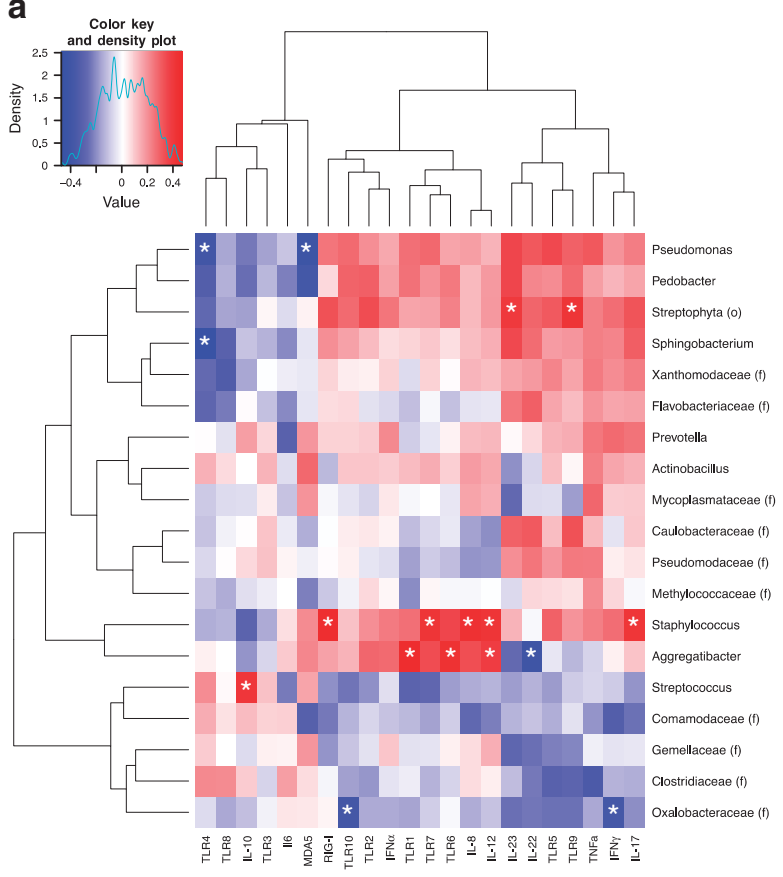

b
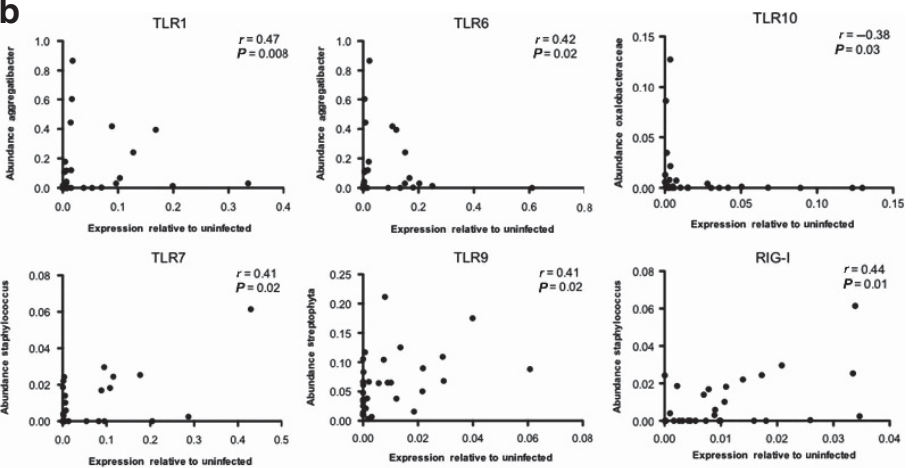

C
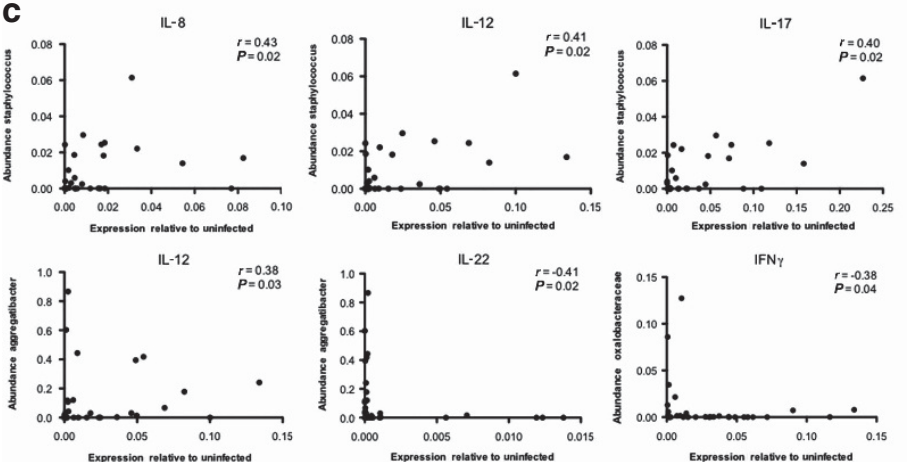

Figure 7 Correlation of the expression of pattern recognition receptors (PRRs) and cytokines with changes in the gut microbiota during simian immunodeficiency virus (SIV) infection. (a) A heatmap of Spearman correlation coefficients was generated for abundance of microbial families and relative expression of PRRs and cytokines in the gut during SIV infection. The red color indicates a positive correlation coefficient, and the blue color represents a negative coefficient. Significant correlations (unadjusted $P$-value $\leqslant 0.05$ ) were indicated with an asterisk. Spearman rank correlations between (b) Toll-like receptor (TLR) and (c) cytokines expression and abundance of the most abundant operational taxonomic units. IFN, interferon; IL, interleukin.

Aggregatibacter abundance was positively correlated with the expression of TLR1, TLR6, and IL-12 but negatively correlated with IL-22 expression. Members of this genus are known to possess virulence factors that inhibit activation and function of immune cells. ${ }^{26}$ Similarly, the abundance of organisms belonging to the genus Staphylococcus positively correlated 
with inflammatory cytokines and PRRs, including IL-8, IL-12, IL-17, TLR7, and RIG-I, and the abundance of Oxalobacteraceae negatively correlated with the expression of IFN $\gamma$ and TLR10 (Figure $7 \mathbf{b}, \mathbf{c}$ ). Our data suggest that shifts in bacterial composition and abundance may influence changes in the expression of PRRs and cytokines in the gut during SIV infection.

\section{DISCUSSION}

A complex interplay exists among immune cells, intestinal epithelium, and microbial communities in the maintenance of gastrointestinal homeostasis. Previous studies have demonstrated that disruption of the gut epithelial barrier during chronic HIV and SIV infections may contribute to microbial translocation, increased T-cell turnover, and systemic immune activation. $^{7,2,4,8,11,27,28}$ These pathogenic changes may be initiated early in infection and be driven by severe loss of mucosal CD $4+\mathrm{T}$ cells. ${ }^{1,29,30}$ Although majority of HIV infections occur through mucosal routes, a significant number of new HIV infections occur following an intravenous exposure of the virus. ${ }^{3,5}$ Rhesus macaques infected with SIV through either mucosal route or intravenous exposure progress to acute and chronic stages of infection and demonstrate similar levels of severe CD4 + T-cell depletion, altered T-cell homeostasis, and increased inflammatory cytokines in the gut. ${ }^{29-32}$ Our study highlights the role of the early innate sensing and response through TLR signaling in SIV infection and potential implications in viral pathogenesis. Our findings suggest that SIV infection causes a rapid upregulation of PRR and cytokine gene expression in the gut mucosa during acute SIV infection. However, these increases are markedly diminished during the chronic viral infection. The PRR and cytokine gene expression were associated with changes in the gut microbiota. Thus we identified a potential link between SIV infection-associated PRR-mediated innate immune modulation and microbial dysbiosis. One of the striking observations in our study was the discordance between the peripheral blood and gut mucosal compartments regarding the induction of PRR and cytokine expression during SIV infection. Cells from the peripheral blood are known to be functionally and phenotypically different from the cells that reside in the gut, and this may affect the differential PRR expression and cytokine responses in the peripheral blood vs. the gut mucosal compartment.

Robust changes in PRR gene expression in the gut during early viral infection can originate from direct engagement of the receptors with PRR ligands, increased pro-inflammatory cytokines, or through altered distribution of mucosal cell populations. ${ }^{33}$ Our findings show induction of massive innate immune response in the gut mucosa during acute SIV infection that was reflected by a robust increase in the expression of TLR7, TLR9, MDA5, and RIG-I and by relatively modest increase in TLR3 expression. In agreement with previously reported studies regarding these PRRs inhibiting HIV or SIV replication, ${ }^{25,34}$ we also found that in vivo expression of RIG-I and TLR3 negatively correlated with the replication of pathogenic SIVmac251 but not with non-pathogenic
SIVmac1A11. Although the induction of RIG-I or TLR3 expression could dampen SIV replication, the virus may disarm the innate immune signaling during chronic SIV infection. Modest changes in TLR3 levels in our study could be possibly due to the whole-tissue analysis, which may mask increased TLR expression localized to a small proportion of responding mucosal cells. Although TLR7 agonist could possibly reactivate latent SIV reservoirs (James Whitney et al. Abstract no. 108, CROI, 2015), we did not find a correlation of TLR7 expression with SIV replication. Engagement of TLR7 was shown to elicit an anergic state in human CD4 + T cells, which contributes to $\mathrm{CD} 4+\mathrm{T}$ cells' hyporesponsiveness. ${ }^{35}$ These observations highlight the need for further investigations prior to applying TLR agonists in HIV cure study. We recently reported an induction of TLR4, TLR7, and TLR8 expression in the gut mucosa during very early SIV infection (at 2.5 days following SIV infection). ${ }^{36}$ This coincided with an increase in IL-1 $\beta$ expression and disruption of epithelial barrier. ${ }^{36}$ Collectively, our data suggest that SIV induces robust TLR expression in the gut immediately following the viral exposure. Thus early mucosal response to the viral infection is driven by the robust TLR expression and may set the stage for the immune dysfunction.

Expression of TLRs 4 in the gut mucosa was decreased during acute SIV infection compared with uninfected animals and remained unchanged during chronic viral infection. As TLR4 is involved in activating innate immunity and HIV-specific cytotoxic T lymphocyte activity, impaired TLR4 expression may adversely affect virus-specific immunity. This is noteworthy as the decreased TLR4 expression in the gut mucosa suggests that it may lead to ineffective anti-viral immune response. Impairment of TLR4 expression could be a direct effect of viral antigens. HIV tat is shown to cause decreased TLR4 expression. ${ }^{37}$ A widespread suppression of genes regulating innate immunity, including lipopolysaccharide (LPS) receptors, CD14, and TLR4, was shown in PBMCs of cynomolgus monkeys during acute SHIV 89.6p infection. ${ }^{38} \mathrm{We}$ also found that TLR8 expression in the gut mucosa was not changed during acute and chronic SIV infection despite the presence of viral loads. TLR8 is known to sense viral singlestranded RNA and induce innate immune response. Decreased TLR8 expression was correlated with increased HIV replication in dendritic cells in vitro. ${ }^{39}$ Interestingly, the modulation of TLR8 signaling is mediated through the complement system and has resulted in enhanced HIV infection, suggesting this as an important viral mechanism of immune evasion. ${ }^{40}$ Thus, the virus may highjack innate immunity in the gut mucosa through suppression of TLR4 and TLR8. Our findings suggest that agonists of these TLRs should be explored for enhancing antiviral immunity.

We found a remarkable increase in TLR2 and TLR5 expression during acute SIV infection in the gut. These data suggest an early encroachment of bacteria into lamina propria and consequent microbial stimulation of intestinal immune cells. Substantial dampening of TLR2 and TLR5 expression during chronic infection was surprising, because acute SIV 
infection is associated with disruption of gut epithelial barriers and increased microbial translocation, indicating a possible negative feedback regulation of TLR2/5 signaling and/or changes of gut microbiota diversity. ${ }^{2,3}$

The increased TLR expression in the gut during acute SIV infection coincided with upregulation of several inflammatory cytokines, including TNF $\alpha$ and IFN $\gamma$, which may contribute to viral control either by killing infected cells or by modulating other immune cells. Following the transition to chronic SIV infection, there was a precipitous decline in the expression of PRRs and cytokines in the gut mucosa. In contrast, the levels of PRRs and cytokines in the peripheral blood remained elevated during chronic SIV infection. Changes in the expression of inflammatory cytokines in the intestinal mucosa during chronic HIV infection have been previously reported. ${ }^{41} \mathrm{We}$ found that antigen-presenting cells from peripheral blood of individuals with chronic HIV infection showed aberrant inflammatory cytokine responses to the commensal bacteria, which was in part owing to elevated expression of TLR2 on these cells. ${ }^{42}$ It is not fully understood why the gut mucosa experiences precipitous dampening of the PRR and cytokine expression during chronic SIV infection despite the ongoing viral replication. First, it is possible that the severe depletion of $\mathrm{CD}^{+}{ }^{+} \mathrm{T}$ cells in the gut and altered T-cell homeostasis may cause changes in the PRR expression. Disruption of the gut epithelium and its renewal during SIV and HIV infections may also impact on the TLR expression. Second, SIV or HIV antigens could inhibit the innate immune response that would enable viral replication and persistence. ${ }^{43}$ Third, during chronic viral infection, changes in the gut microbiota composition may downmodulate the expression of PRRs (Figure 7) and the associated inflammatory cytokines. Reduced PRR expression during chronic SIV infection may compromise the ability of gut immune cells to sense and respond to the virus itself as well as to other pathogens, while dampening of PRR and cytokine expression in the gut may limit the gut tissue damage caused by chronic inflammation.

We found that the initiation of ART during early SIV infection resulted in preservation of higher PRR and cytokine expression in the gut (Figures $\mathbf{2 b}$ and $\mathbf{4 b}$ ) and led to effective suppression of viral replication, high recovery of $\mathrm{CD} 4{ }^{+} \mathrm{T}$ cells, and induction of mucosal repair/renewal-associated gene expression in the gut as compared with those initiating ART during chronic infection. ${ }^{20,23}$ However, early ART failed to completely suppress viral replication or eradicate the virus. Lack of total recovery of the gut mucosal immune compartment and PRR expression may be reflective of residual viral replication and immune activation as well as microbial translocation in the mucosa. Previous report indicates that very early ART in the acute SIV/HIV infection has limited effect on eradication of viral reservoirs, which may be associated with dampened PRR-cytokine signaling. ${ }^{44}$

The non-pathogenic SIVmac1A11 successfully establishes infection in the host but does not cause immunodeficiency. ${ }^{21,22}$ The acute SIV1A11 infection elicited an increase in PRR and cytokine gene expression levels in the gut that was similar to the increase observed during acute SIVmac251 infection. However, PRR and cytokine expression levels decreased during chronic SIVmac1A11 infection, which were similar to those in uninfected controls. Previously, we found that dissemination and tissue localization of pathogenic SIVmac251 was very different from non-pathogenic SIVmac1A11, which may be associated with increased migration of inflammatory cells into the intestinal mucosa. ${ }^{45}$ Together, these findings suggest that, unlike pathogenic SIVmac251, infection with non-pathogenic SIVmac1A11 might not cause epithelial damage and microbiota translocation. Alternatively, SIVmac1A11-induced gut epithelial damage might be repaired during chronic viral infection.

We found that gut microbiota from SIV-infected macaques showed decreased abundance of Firmicutes and increased abundance of Proteobacteria. Similar findings have been previously reported in HIV-infected patients. ${ }^{11-13,16}$ Administration of sevelamer, which binds to microbial LPS, reduced immune activation in SIV infected macaques. ${ }^{46}$ However, sevelamer failed to decrease circulating LPS or soluble CD14 levels in ART-naive HIV-infected patients. ${ }^{47}$ These findings suggest that blocking of LPS in circulation alone was not sufficient to completely resolve immune activation. Thus further investigations are needed to develop strategies to reverse changes in the gut microbiota during pathogenic infections.

To date, most studies have examined colon contents and fecal samples for assessing changes in gut microbiota. However, recent studies have clearly shown that composition and diversity of microbiota in various parts of the gastrointestinal tract (including small and large intestine) are somewhat different. These differences may be driven by the local microenvironment that may differ in its $\mathrm{pH}$, oxygen, carbon, ions and minerals and microbial exposures. Our study focused on the small intestine where the majority of the terminal nutrient digestion and absorption occurs and HIV and SIV infections cause gut epithelial barrier disruption, nutrient malabsorption, and severe CD4 + T-cell depletion. ${ }^{1,4,27,29,30,32}$ We examined changes in the gut microbiota from the small intestine in order to investigate correlations of the mucosal TLR expression and inflammation with gut microbiota changes at the same local site in the gut microenvironment.

Altered microbiota composition could be responsible for changes in PRR expression in the gut, leading to increased recognition of the commensal microbiota, resulting in progressive inflammation and dysbiosis. Alternatively, the increased expression of PRR and inflammatory cytokines could mediate changes of the microbiota composition, also resulting in progressive inflammation and dysbiosis. Consistent with this possibility, an increased prevalence of Proteobacteria has been observed in intestinal inflammation, because the inflamed gut provides new sources of nutrients for bacterial species within this phylum. ${ }^{48}$ Furthermore, PRR signaling could be positively or negatively selected for specific taxa. ${ }^{49}$ In this regard, we observed several associations between gut microbiota and PRR and cytokine expression. For example, Aggregatibacter, which has been previously shown to induce TLR2 expression, was 
positively associated with TLR1, TLR6, and IL-12 expression (Figure $\mathbf{7 b}, \mathbf{c}$ ). It is known that TLR2 signaling occurs via heterodimerization with either TLR1 or TLR6 and that heterodimers can sense a wide range of molecules mainly not only from Gram-positive but also from Gram-negative bacteria, generating an inflammatory milieu. ${ }^{33}$ The increased abundance of Aggregratibacter coupled with increased TLR1/2/ 6 expression suggests a link between local inflammation and dysbiosis during acute SIV infection. Moreover, the abundance of Staphylococcus correlates with several cytokines and PRR. These results are also consistent with previous reports that highlight the ability of members of this genus to induce the production of inflammatory cytokines. ${ }^{50}$

Our study captures the in vivo gut mucosal response to SIV infection and highlights changes in PRR and cytokine gene expression, as well as their potential effects on the resident gut microbiota. Early ART partially restored PRR and cytokine expression in the gut. Future studies on the functionality of individual cell subsets such as macrophages, dendritic cells, or intestinal epithelial cells will help define their role in the innate gut immune response during early infection. A better understanding of HIV-induced PRR signaling and its impact on the innate immune responses is critical to the development of improved therapeutic strategies for viral suppression and enhanced immune recovery.

\section{METHODS}

Animal subjects, infection, and sample collection. The study involved a cross-sectional analysis of samples of jejunum and peripheral blood mononuclear cells from rhesus macaques infected intravenously with either pathogenic SIVmac251 or non-pathogenic SIVmac1A11 (see Supplementary Table S1). Tissue samples were collected at necropsy, and immediately cryopreserved $-80^{\circ} \mathrm{C}$, during acute (at 1 and 2 weeks following SIVmac251 or SIVmac1A11 infection) or chronic stages (at 10-34 weeks post-SIVmac251 infection and at 8-23 weeks post-SIV1A11 infection) of SIV infection as well as from SIV-negative healthy controls. Tissue samples were also obtained from rhesus macaques that started receiving combination ART (PMPA and FTC) at 1 week post-SIVmac251 infection and continued for 30 weeks thereafter as previously reported. $^{20}$

Cell isolation and flow cytometry. PBMCs from peripheral blood and lamina propria lymphocytes from gut tissue samples were isolated as previously described. ${ }^{31,36}$ Flow cytometric analysis was performed to assess the prevalence of $\mathrm{CD}^{+}{ }^{+} \mathrm{CD} 4{ }^{+} \mathrm{T}$ cells using $\mathrm{T}$-cell phenotype markers CD3 (SP34-2, BD Bioscience) and CD4 (OKT4, eBioscience, San Jose, CA) as previously described. ${ }^{36}$ Isolated PBMCs and gut tissue samples were flash frozen in liquid nitrogen and cryopreserved for further analysis.

RNA isolation and gene expression analysis. In all, $50 \mathrm{mg}$ of frozen tissues and pelleted cryopreserved PBMCs were used for RNA extraction using the RNeasy Kit (Qiagen, Valencia, CA). Quantitative real-time PCR was performed to measure RNA levels of TLR and cytokine genes as previously reported. ${ }^{28}$ Levels of gene transcription were normalized to glyceraldehyde 3-phosphate dehydrogenase or $\beta$-actin, and data were expressed as a relative fold change from uninfected controls (see Supplementary Information). SIV RNA loads in plasma and gut tissue samples were determined by real-time PCR assay as described previously. ${ }^{31,36}$
Gut microbiota analysis. DNA was isolated from jejunal tissue samples using the PowerSoil Kit protocol (MoBio), amplified using a nested PCR protocol and barcoded primer specific for V4 region of $16 \mathrm{~S}$ rRNA gene, and quantified. Sequences were generated (MoBio, Carlsbad, CA) and (MiSeq Sequencer, Illumina, San Diego, CA). filtered for quality, and analyzed using the QIIME software (Flagstaff, AZ). Details are provided in the Supplementary Information.

Statistical analysis. Statistical differences between groups were analyzed using analysis of variance with Tukey's post-test on normalized $\mathrm{Ct}$ values. Spearman correlation coefficients were determined by calculating using normalized Ct values. Differences in microbial abundance were analyzed using non-parametric Mann-Whitney $U$-tests. Correlation analysis and heatmaps were performed with the R Statistical Software.

SUPPLEMENTARY MATERIAL is linked to the online version of the paper at http://www.nature.com/mi

\section{ACKNOWLEDGMENTS}

We thank Dr Charles Bevins and Dr Patricia Castillo for their design of the nested PCR protocol. This work was supported by the UC Davis RISE grant and $\mathrm{NIH}$ grants Al43274 and DK61297 to S.D. and by a postdoctoral fellowship from the Brazilian funding agency CNPq to C.S.R. We thank Beau Parry for help in manuscript preparation.

\section{AUTHOR CONTRIBUTIONS}

Conceived and designed the experiments: T.W.G. and S.D. Performed the experiments: T.W.G., C.A.G., C.S.R., S.S.-W., L.A.H., and M.R. Analyzed the data: T.W.G., C.S.R., C.A.G., L.R.G., A.J.B., G.J., and S.D. Contributed to the writing of the manuscript: T.W.G., G.J., C.S.R., C.A.G., L.A.H., and S.D.

\section{DISCLOSURE}

The authors declared no conflict of interest.

c 2016 Society for Mucosal Immunology

\section{REFERENCES}

1. Guadalupe, M. et al. Severe CD4 + T-cell depletion in gut lymphoid tissue during primary human immunodeficiency virus type 1 infection and substantial delay in restoration following highly active antiretroviral therapy. J. Virol. 77, 11708-11717 (2003).

2. Moir, S., Chun, T.W. \& Fauci, A.S. Pathogenic mechanisms of HIV disease. Annu. Rev. Pathol. 6, 223-248 (2011).

3. Dandekar, S., George, M.D. \& Baumler, A.J. Th17 cells, HIV and the gut mucosal barrier. Curr. Opin. HIV AIDS 5, 173-178 (2010).

4. Sankaran, S. et al. Rapid onset of intestinal epithelial barrier dysfunction in primary human immunodeficiency virus infection is driven by an imbalance between immune response and mucosal repair and regeneration. J. Virol. 82, 538-545 (2008).

5. Maartens, G., Celum, C. \& Lewin, S.R. HIV infection: epidemiology, pathogenesis, treatment, and prevention. Lancet 384, 258-271 (2014).

6. Guadalupe, M. et al. Viral suppression and immune restoration in the gastrointestinal mucosa of human immunodeficiency virus type 1-infected patients initiating therapy during primary or chronic infection. J. Virol. 80, 8236-8247 (2006).

7. Klatt, N.R., Funderburg, N.T. \& Brenchley, J.M. Microbial translocation, immune activation, and HIV disease. Trends Microbiol. 21, 6-13 (2013).

8. Deeks, S.G. et al. Immune activation set point during early HIV infection predicts subsequent CD4 + T-cell changes independent of viral load. Blood 104, 942-947 (2004).

9. Keedy, K.S. \& Margolis, D.M. Therapy for persistent HIV. Trends Pharmacol. Sci. 31, 206-211 (2010).

10. Macal, M. et al. Effective CD4+ T-cell restoration in gut-associated lymphoid tissue of HIV-infected patients is associated with enhanced Th17 
cells and polyfunctional HIV-specific T-cell responses. Mucosal Immunol. 1, 475-488 (2008).

11. Vujkovic-Cvijin, I. et al. Dysbiosis of the gut microbiota is associated with HIV disease progression and tryptophan catabolism. Sci. Transl. Med. 5, 193ra191 (2013).

12. Mutlu, E.A. et al. A compositional look at the human gastrointestinal microbiome and immune activation parameters in HIV infected subjects. PLoS Pathog. 10, e1003829 (2014).

13. Lozupone, C.A. et al. Alterations in the gut microbiota associated with HIV1 infection. Cell Host Microbe 14, 329-339 (2013).

14. Brestoff, J.R. \& Artis, D. Commensal bacteria at the interface of host metabolism and the immune system. Nat. Immunol. 14, 676-684 (2013).

15. Manichanh, C., Borruel, N., Casellas, F. \& Guarner, F. The gut microbiota in IBD. Nat. Rev. Gastroenterol. Hepatol. 9, 599-608 (2012).

16. Dillon, S.M. et al. An altered intestinal mucosal microbiome in HIV-1 infection is associated with mucosal and systemic immune activation and endotoxemia. Mucosal Immunol. 7, 983-994 (2014).

17. Klase, Z. et al. Dysbiotic bacteria translocate in progressive SIV infection. Mucosal Immunol. 8, 1009-1020 (2015).

18. Ocon, S. et al. Transcription profiling reveals potential mechanisms of dysbiosis in the oral microbiome of rhesus macaques with chronic untreated SIV infection. PLoS One 8, e80863 (2013).

19. Moeller, A.H. et al. SIV-induced instability of the chimpanzee gut microbiome. Cell Host Microbe 14, 340-345 (2013).

20. Verhoeven, D., Sankaran, S., Silvey, M. \& Dandekar, S. Antiviral therapy during primary simian immunodeficiency virus infection fails to prevent acute loss of CD4 $+\mathrm{T}$ cells in gut mucosa but enhances their rapid restoration through central memory Tcells. J. Virol. 82, 4016-4027 (2008).

21. Marthas, M.L. et al. Rhesus macaques inoculated with molecularly cloned simian immunodeficiency virus. J. Med. Primatol. 18, 311-319 (1989).

22. Lackner, A.A., Vogel, P., Ramos, R.A., Kluge, J.D. \& Marthas, M. Early events in tissues during infection with pathogenic (SIVmac239) and nonpathogenic (SIVmac1A11) molecular clones of simian immunodeficiency virus. Am. J. Pathol. 145, 428-439 (1994).

23. George, M.D., Reay, E., Sankaran, S. \& Dandekar, S. Early antiretroviral therapy for simian immunodeficiency virus infection leads to mucosal CD4 + T-cell restoration and enhanced gene expression regulating mucosal repair and regeneration. J. Virol. 79, 2709-2719 (2005).

24. Wang, Y., Wang, X., Li, J., Zhou, Y. \& Ho, W. RIG-I activation inhibits HIV replication in macrophages. J. Leukoc. Biol. 94, 337-341 (2013).

25. Buitendijk, M., Eszterhas, S.K. \& Howell, A.L. Toll-like receptor agonists are potent inhibitors of human immunodeficiency virus-type 1 replication in peripheral blood mononuclear cells. AIDS Res. Hum. Retroviruses 30, 457-467 (2014).

26. Fives-Taylor, P.M., Meyer, D.H., Mintz, K.P. \& Brissette, C. Virulence factors of Actinobacillus actinomycetemcomitans. Periodontology 2000 20, 136-167 (1999).

27. Sankaran, S. et al. Gut mucosal T cell responses and gene expression correlate with protection against disease in long-term HIV-1-infected nonprogressors. Proc. Natl. Acad. Sci. USA 102, 9860-9865 (2005).

28. Raffatellu, M. et al. Simian immunodeficiency virus-induced mucosal interleukin-17 deficiency promotes Salmonella dissemination from the gut. Nat. Med. 14, 421-428 (2008).

29. Veazey, R.S. et al. Gastrointestinal tract as a major site of CD4 + T cell depletion and viral replication in SIV infection. Science 280, 427-431 (1998).

30. Smit-McBride, Z., Mattapalli, J.J., McChesney, M., Ferrick, D. \& Dandekar, S. Gastrointestinal T lymphocytes retain high potential for cytokine responses but have severe $\mathrm{CD} 4(+) \mathrm{T}$-cell depletion at all stages of simian immunodeficiency virus infection compared to peripheral lymphocytes. J. Virol. 72, 6646-6656 (1998).

31. Verhoeven, D. et al. Enhanced innate antiviral gene expression, IFN-alpha, and cytolytic responses are predictive of mucosal immune recovery during simian immunodeficiency virus infection. J. Immunol. 192, 3308-3318 (2014).

32. Heise, C., Vogel, P., Miller, C.J., Halsted, C.H. \& Dandekar, S. Simian immunodeficiency virus infection of the gastrointestinal tract of rhesus macaques. Functional, pathological, and morphological changes. Am. J. Pathol. 142, 1759-1771 (1993).

33. Brubaker, S.W., Bonham, K.S., Zanoni, I. \& Kagan, J.C. Innate immune pattern recognition: a cell biological perspective. Annu. Rev. Immunol. 33, 257-290 (2015).

34. Sang, M., Liu, J.B., Dai, M., Wu, J.G. \& Ho, W.Z. Toll-like receptor 3 signaling inhibits simian immunodeficiency virus replication in macrophages from rhesus macaques. Antiviral Res. 112, 103-112 (2014).

35. Dominguez-Villar, M., Gautron, A.S., de Marcken, M., Keller, M.J. \& Hafler, D.A. TLR7 induces anergy in human CD4(+) T cells. Nat. Immunol. 16, 118-128 (2015).

36. Hirao, L.A. et al. Early mucosal sensing of SIV infection by paneth cells induces IL-1beta production and initiates gut epithelial disruption. PLOS Pathog. 10, e1004311 (2014).

37. Ben Haij, N. et al. HIV-1 tat protein induces production of proinflammatory cytokines by human dendritic cells and monocytes/macrophages through engagement of TLR4-MD2-CD14 complex and activation of NF-kappaB pathway. PLoS One 10, e0129425 (2015).

38. Bosinger, S.E. et al. Global genomic analysis reveals rapid control of a robust innate response in SIV-infected sooty mangabeys. J. Clin. Invest. 119, 3556-3572 (2009).

39. Gringhuis, S.I. et al. HIV-1 exploits innate signaling by TLR8 and DC-SIGN for productive infection of dendritic cells. Nat. Immunol. 11, 419-426 (2010).

40. Ellegard, R. et al. Complement opsonization of HIV-1 results in decreased antiviral and inflammatory responses in immature dendritic cells via CR3. J. Immunol. 193, 4590-4601 (2014).

41. McGowan, I., Radford-Smith, G. \& Jewell, D.P. Cytokine gene expression in HIV-infected intestinal mucosa. Aids 8, 1569-1575 (1994).

42. Nagy, L.H. et al. Chronic HIV infection enhances the responsiveness of antigen presenting cells to commensal Lactobacillus. PLoS One 8, e72789 (2013).

43. Mogensen, T.H., Melchjorsen, J., Larsen, C.S. \& Paludan, S.R. Innate immune recognition and activation during $\mathrm{HIV}$ infection. Retrovirology 7, 54 (2010).

44. Whitney, J.B. et al. Rapid seeding of the viral reservoir prior to SIV viraemia in rhesus monkeys. Nature 512, 74-77 (2014).

45. Stone, J.D., Heise, C.C., Canfield, D.R., Elices, M.J. \& Dandekar, S. Differences in viral distribution and cell-adhesion molecule expression in the intestinal-tract of rhesus macaques infected with pathogenic and nonpathogenic SIV. J. Med. Primatol. 24, 132-140 (1995).

46. Kristoff, J. et al. Early microbial translocation blockade reduces SIV-mediated inflammation and viral replication. J. Clin. Invest. 124, 2802-2806 (2014).

47. Sandler, N.G. et al. Sevelamer does not decrease lipopolysaccharide or soluble CD14 levels but decreases soluble tissue factor, low-density lipoprotein (LDL) cholesterol, and oxidized LDL cholesterol levels in individuals with untreated HIV infection. J. Infect. Dis. 210, 1549-1554 (2014).

48. Winter, S.E., Lopez, C.A. \& Baumler, A.J. The dynamics of gut-associated microbial communities during inflammation. EMBO Rep. 14, 319-327 (2013).

49. Round, J.L. et al. The Toll-like receptor 2 pathway establishes colonization by a commensal of the human microbiota. Science 332 , 974-977 (2011).

50. Edwards, L.A. et al. Enterotoxin-producing staphylococci cause intestinal inflammation by a combination of direct epithelial cytopathy and superantigen-mediated T-cell activation. Inflamm. Bowel Dis. 18, 624-640 (2012). 